\title{
Gap junctions in the cardiovascular and immune systems
}

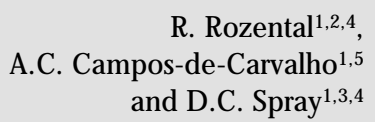

R. Rozental ${ }^{1,2,4}$

A.C. Campos-de-Carvalho ${ }^{1,5}$ and D.C. Spray1,3,4

\author{
Departments of ${ }^{1} \mathrm{~N}$ euroscience, ${ }^{2} \mathrm{Anesthesiology}$ and ${ }^{3} \mathrm{M}$ edicine, \\ Albert Einstein College of Medicine, Bronx, NY, USA \\ ${ }^{4}$ Departamento de Medicina Interna, Instituto da Visão and IPTESP, \\ Universidade Federal de Goiás, Goiania, GO, Brasil \\ Instituto de Biofísica "Carlos Chagas Filho", \\ Universidade Federal do Rio de Janeiro, Rio de Janeiro, RJ, Brasil
}

\section{Correspondence \\ R. Rozental \\ Department of Neuroscience Albert Einstein College of Medicine Bronx, NY 10461 \\ USA \\ O verview about the Meeting "Gap Junctions in the Nervous and Cardiovascular Systems: Clinical Implications", Rio de Janeiro, RJ, Brazil, June 6-11, 1998.}

\begin{abstract}
Gap junctions are clusters of intercellular channels directly connecting the cytoplasm of adjacent cells. These channels are formed by proteins named connexins and are present in all metazoan organisms where they serve diverse functions ranging from control of cell growth and differentiation to electric conduction in excitable tissues. In this overview we describe the presence of connexins in the cardiovascular and lympho-hematopoietic systems giving the reader a summary of the topics to be covered throughout this edition and a historical perspective of the discovery of gap junctions in the immune system.
\end{abstract}

Key words

- Gap junctions

- Cardiovascular system

- Immune system

- Connexins

\section{Gap junction channels: A simple overview}

Gap junctions are clusters of intercellular channels providing conduits for diffusional exchange of ions and small molecules directly from one cell to another (for details, see Ref. 1). These channels span two plasma membranes and result from the association of two half channels, termed connexons, contributed separately by each cell of a pair (see Figure 1). Connexins are expressed in an overlapping pattern of tissue distribution and more than one connexin type is generally expressed by individual cells. In addition, the hemichannels or connexons contributed by each cell may have selective affinities for one another either within or between connexins, forming homotypic, heterotypic and heteromeric junctions (Figure 1; see also Brink et al. (p. 415), Verselis et al. (p. 379) and Beyer et al. (p. 391) in this edition). A homotypic gap junction channel is composed of 12 identical connexin subunits; in heterotypic but homomeric channels all the connexins in the connexon of one cell are identical but are different from the connexins of the adjacent cell. In heteromeric gap junction channels, the connexins comprising the connexon are not identical. The expression of numerous connexin types in the cardiovascular and immune system provides an enormous diversity of possible channel configurations; whether some are favored for some functions (as might be the case for homomeric heterotypic junctions at compartmental boundaries) remains to be established.

Characterization of the properties of gap junctions formed by individual connexin types (and how these properties are changed in heterotypic pairings) has been a major effort of several laboratories. Although the 
biophysical and biochemical characterization of these channels lags behind the understanding of other voltage- or ligand-gated ion channel complexes, recent application of molecular genetic approaches has had a major impact. In this edition, for example, Nicholson et al. (p. 369) discuss the molecular basis of selective permeability between gap junction channels composed of different connexins and Yahuaca et al. (p. 399) present their results on phosphorylation of $\mathrm{Cx} 43$.

\section{Gap junctions in the heart and vasculature}

Gap junctions in the heart mediate the intercellular transmission of electrical impulses that coordinate the generation of the cardiac rhythm, conduct the pacemaking signal to muscular regions and synchronize contractions of the atrium and ventricles. The three most abundant cardiac gap junction proteins are $\mathrm{Cx} 43, \mathrm{Cx} 40$, and $\mathrm{Cx} 45$, which are expressed within each region of the heart to different extents and in various combinations. For example, $\mathrm{Cx} 43$ is most abundant in the ventricle, $\mathrm{Cx} 40$ in the atrium, and $\mathrm{Cx} 40$ and $\mathrm{Cx} 45$ in the conduction system (2).

Because the major role of gap junctions in the normal heart is the generation and maintenance of rhythmic contractions, considerable recent work has been directed at

Figure 1 - Possible configurations of gap junction channels which are formed by the end to end (here, top to bottom) alignment of two connexons; two differently shaded components of connexons represent the two connexin subunits.
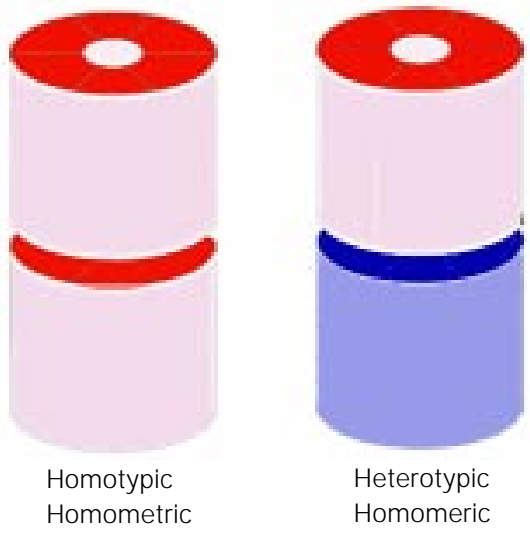

understanding how altered gap junction distribution and function may lead to disturbed cardiac rhythmicity, including reentry. For example, in aging and post-infarct myocardium, gap junction disappearance and remodeling accentuate conduction discontinuities, as summarized in the manuscript in this issue by Jeff Saffitz (p. 407). Moreover, ventricular conduction in $\mathrm{Cx} 43$ heterozygous mice (lacking one copy of the $\mathrm{Cx} 43$ gene) is significantly slowed (3); atrial conduction time is prolonged (and supraventricular arrhythmias occasionally appear) in Cx40 knockout mice $(4,5)$.

A disease of major importance to Brazil and other countries in Latin America is chagasic cardiomyopathy, which is characterized by both acute and chronic phases; it affects the cardiac system (Figure 2), the smooth muscles and the nervous system. Of particular interest, acute infection of cardiac myocytes and CNS glia results in loss of intercellular communication (Figure 2) without affecting expression levels or post-translational processing of gap junction proteins $(6,7)$. It appears as if the changes in gap junction distribution result from altered trafficking of the junctional proteins to or from the plasma membrane, but the mechanisms remain to be resolved. Masuda et al. (p. 439) in their paper in this collection present a novel set of findings in this area, showing that sera from chagasic patients block inter- 
cellular communication and atrioventricular conduction in cardiac myocytes. Thus, perhaps the protozoan parasite appears to exert both local and long-range disorganization of $\mathrm{Cx} 43$, generating arrhythmias and thereby disturbing cardiac function.

Gap junctions in the vessel wall play critical roles in maintenance and modulation of vascular tone. In this issue, George Christ and colleagues (p. 423) summarize the evidence for this role of gap junctions between smooth muscle cells, indicating that these channels provide the critical pathway for second messenger diffusion in this process. Endothelial cells are also interconnected by gap junctions, and this pathway presumably serves to coordinate vascular tone through coordinated nitric oxide production and distribution. In the vessels of hypertensive rats, Haefliger and Meda (p. 431) show that chronic hypertension alters the expression of $\mathrm{Cx} 43$ in muscle cells; they suggest that the cell-cell communication mediated by $\mathrm{Cx} 43$ may contribute to regulate the elasticity of the vascular wall.

\section{Immune response}

The roles of gap junction channels in the immune system is increasingly appreciated. As early as 30 years ago electron microscopy and functional studies revealed close contacts between lymphoreticular cells in rheumatoid synovial membrane and suggested the expression of gap junction channels among activated macrophages (8-10).

Although the argument can be traced earlier, the modern portion of the story begins with Gilberto M. Oliveira Castro and Marcelo A. Barcinski, at that time Associate Professors at the Institute of Biophysics "Carlos Chagas Filho" at the Federal University of Rio de Janeiro. Of special interest, Oliveira Castro collaborated with and was highly influenced by the work of Werner R. Loewenstein (11) in their initial studies of the effects of divalent cations on junctional membrane permeability. These studies were further developed in Brazil and, together with the work by Marcelo A. Barcinski, they showed the existence of intercellular communication in stimulated human lymphocytes (12) and suggested that calcium could mediate uncoupling of these cells (13). Such collaborations were very fruitful in the field of immunology and their major results are well known to everyone in this field. Furthermore, this work impacted on different generations of electrophysiologists at the Institute of Biophysics, among them Antonio C. Campos de Carvalho, Pedro Persechini and George A. Dos Reis, who have characterized, among other issues, the ATP-induced currents in macrophages and electrophysiological properties of phagocytic membranes in activated macrophages (14-17).

The occurrence of gap junctions in mi-

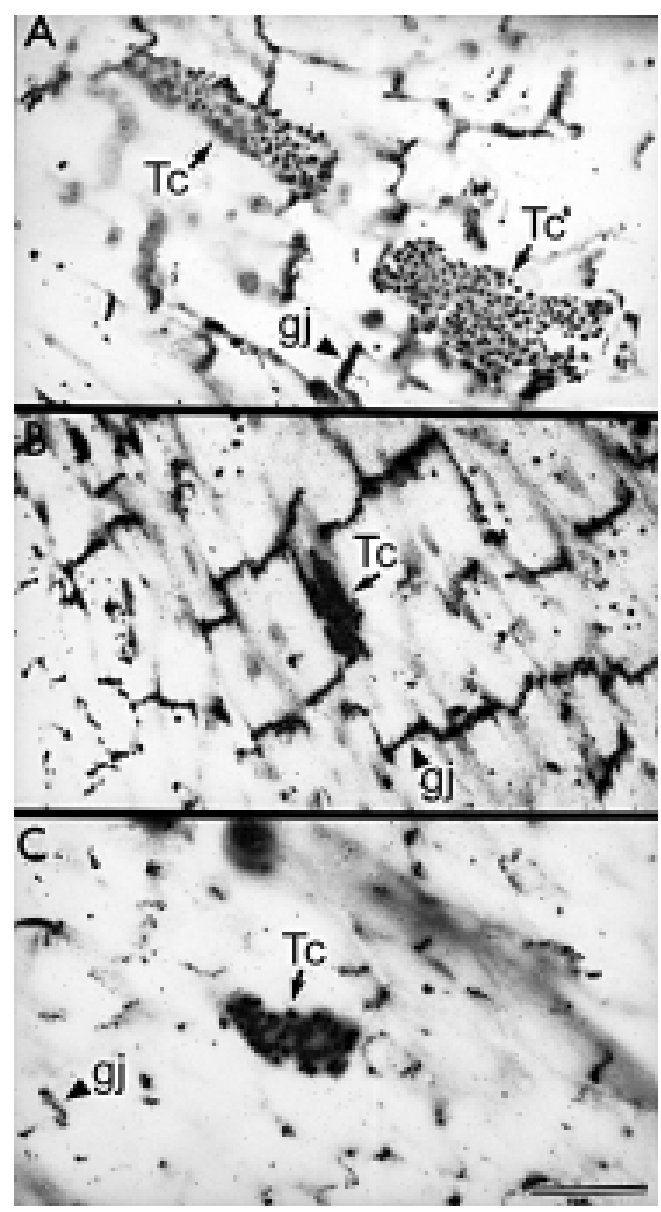

Figure 2 - Disruption of intercalated disks in hearts of mice infected with Trypansoma cruzi, the protozoan parasite that causes Chagas' disease. A, B and $C$ illustrate sections of $T$. cruzi-infected mouse heart immunostained with antibody specific for Cx43. Note the presence of normal-appearing intercalated disks (gap junctions: $g$ j, arrows) in uninfected myocytes and their disruption in cells infected with T. cruzi (Tc, arrows). Frozen sections and immunostaining performed by C. Roy; infected mouse hearts obtained from Dr. H.B. Tanowitz. Bar = $100 \mu \mathrm{m}$. 
gratory cells (i.e., macrophages) of the immune system has been a controversial issue. Nevertheless, gap junctions composed of Cx43 have been identified in blood-forming tissue and in hemopoietic cultured cells (e.g., lymphocytes). At the Rio de Janeiro meeting, Rosendall presented data on the functional coupling between follicular dendritic cells and lymphocytes and suggested that metabolic cooperation between these cells is important to time germinal center events. Furthermore, Rosendall suggested that second messenger permeation through gap junction channels may be involved in the rescue of B cells from apoptotic cell death. In this edition, Sáez et al. (p. 447) and Alves et al. (p. 457) discuss the involvement of gap junction channels in the response of the immune system and the effects of different neuropeptides and hormones on the modulation of intercellular communication in thymic cells.

\section{Acknowledgments}

Secretarial and editorial assistance with the manuscript was provided by Ms. Fran Andrade. We thank Mr. Alfredo Fort for preparing Figure 1.

\section{References}

1. Spray DC, Scemes E \& Rozental R (1998). Cell-cell communication via gap junctions. In: Zigmond MJ, Bloom FE, Landis SC, Roberts J L \& Squire LR (Editors), Fundamental Neuroscience. Academic Press, New York, 317-343.

2. Spray DC, Suadicani SO \& Srinivas M (2000). In: Sperelakis N \& Kurachi Y (Editors), Gap J unctions in the Heart. Academic Press, New York (in press).

3. Thomas SA, Schuessler RB, Berul $\mathrm{Cl}$, Beardslee MA, Beyer EC, Mendelsohn ME \& Saffitz J E (1998). Disparate effects of deficient expression of connexin43 on atrial and ventricular conduction: evidence for chamber-specific molecular determinants of conduction. Circulation, 97: 686691.

4. Simon AM, Goodenough DA \& Paul DL (1998). Mice lacking connexin40 have cardiac conduction abnormalities characteristic of atrioventricular block and bundle branch block. Current Biology, 8: 295-298.

5. Hagendorff A, Schumacher B, Kirchhoff S, Luderitz B \& Willecke K (1999). Conduction disturbances and increased atrial vulnerability in connexin40-deficient mice analyzed by transesophageal stimulation. Circulation, 99: 1508-1515.

6. Campos de Carvalho AC, Tanowitz H, Wittner $M$, Dermietzel R, Roy C, Hertzberg EL \& Spray DC (1992). Gap junction distribution is altered between cardiac myocytes infected with Trypanosoma cruzi. Circulation Research, 70: 733742.

7. Campos de Carvalho AC, Roy C, Hertzberg EL, Tanowitz HB, Kessler J A, Weiss LM, Wittner M, Dermietzel R, Gao Y \& Spray DC (1998). Gap junction disappearance in astrocytes and leptomeningeal cells as a consequence of protozoan infection. Brain Research, 790: 304-314.

8. Neumark T (1977). Cell-to-cell contacts between lymphoreticular cells in rheumatoid synovial membrane. Acta Morphologica Academiae Scientiarum Hungaricae, 25: 121-135.

9. Sellin D, Wallach DF \& Fischer H (1971). Intercellular communication in cell-mediated cytotoxicity. Fluorescein transfer between $\mathrm{H}-2 \mathrm{~d}$ target cells and $\mathrm{H}-2 \mathrm{~b}$ lymphocytes in vitro. European J ournal of Immunology, 1: 453-458.

10. Hulser DF \& Peters JH (1972). Contact cooperation in stimulated lymphocytes. II. Electrophysiological investigations on intercellular communication. Experimental Cell Research, 74: 319-326.

11. Oliveira-Castro GM \& Loewenstein WR (1971). J unctional membrane permeability: Effects of divalent cations. J ournal of Membrane Biology, 5: 51-77.

12. Oliveira-Castro GM, Barcinski MA \& Cukierman S (1973). Intercellular communication in stimulated human lympho- cytes. J ournal of Immunology, 111: 16161619.

13. Oliveira-Castro GM \& Barcinski MA (1974). Calcium-induced uncoupling in communicating human lymphocytes. Biochimica et Biophysica Acta, 352: 338-343.

14. Albuquerque $C$, Oliveira SM, CoutinhoSilva R, Oliveira-Castro GM \& Persechini PM (1993). ATP- and UTP-induced currents in macrophages and macrophage polykaryons. American J ournal of Physiology, 265: C1663-C1673.

15. Persechini PM \& Oliveira-Castro GM (1987). Electrophysiology of phagocytic membranes: intracellular $\mathrm{K}^{+}$activity and $\mathrm{K}^{+}$equilibrium potential in macrophage polykaryons. Biochimica et Biophysica Acta, 899: 213-221.

16. Dos Reis GA, Persechini PM, Ribeiro J M \& Oliveira-Castro GM (1979). Electrophysiology of phagocytic membranes. II. Membrane potential and induction of slow hyperpolarizations in activated macrophages. Biochimica et Biophysica Acta, 552: 331-340.

17. Oliveira-Castro GM \& Dos Reis GA (1981). Electrophysiology of phagocytic membranes. III. Evidence for a calcium-dependent potassium permeability change during slow hyperpolarizations of activated macrophages. Biochimica et Biophysica Acta, 640: 500-511. 